\title{
Construction of Periodic Solutions of One Class Nonautonomous Systems of Differential Equations
}

\author{
Alexander N. Pchelintsev \\ Tambov State Technical University, Tambov, Russia \\ Email: pchelintsev.an@yandex.ru
}

Received June 15, 2013; revised July 16, 2013; accepted August 1, 2013

Copyright (C) 2013 Alexander N. Pchelintsev. This is an open access article distributed under the Creative Commons Attribution License, which permits unrestricted use, distribution, and reproduction in any medium, provided the original work is properly cited.

\begin{abstract}
In this article we proposed a method for constructing approximations to periodic solutions of one class nonautonomous system of ordinary differential equations. It is based on successive approximation scheme using parallel symbolic calculations to obtain solutions in analytical form. We showed the convergence of the scheme of successive approximations on the period, and also considered an example of a second order system where the described scheme of calculations can be applied.
\end{abstract}

Keywords: Periodic Solution; System of Ordinary Differential Equations; Scheme of Successive Approximations; Symbolic Calculations

\section{Introduction}

Quite often (and in practice as well) there appears a problem of constructing of periodic solutions of the normal system of ordinary differential equations of the form

$$
\dot{x}=f(t, x),
$$

where $x(t)$-vector function of a real variable $t, f(t, x)$ -vector function equal to

$$
f(t, x)=\varphi(x)+h(t),
$$

where vector $\varphi(x)$-multidimensional polynomial and function $h(t)$ is a trigonometric polynomial (T-periodic vector function).

Many of the theorems [1] of existence of periodic solutions of system (1) use the fundamental fact that such solutions are completely determined by the fixed points of the shift operator along the trajectories of the system. These theorems can not be used to direct finding of the desired periodic solution.

Let it be known that the system (1) has a unique $T$-periodic solution $x^{*}(t)$. Examples of systems that have a unique periodic solution, are the systems with convergence [2,3]. In this article one class of such systems would be considered so that for them we provide a method of constructing of approximations to the solution $x^{*}(t)$; given the conditions imposed on the function $f$, it allows showing the convergence of the scheme of successive approximations on the period. At that it introduces an auxil- iary system for constructing in a symbolic form of approximation to some periodic function, which depends on the initial conditions for the system (1). By varying those conditions we will find an approximation to the solution $x^{*}(t)$. The parallel calculations can be used to improve efficiency of calculation process. The symbolic form of representation is convenient because it further allows you to analyze the harmonic components of the desired approximation.

\section{The Conditions Imposed on Original System}

We shall consider the class of systems (1), which obeys the following conditions:

1) The system (1) is a system with convergence, i.e., it has a unique $T$-periodic solution $x^{*}(t)$, that asymptotically stable in whole.

2) Closed ball $S_{r}$ of radius $r$, which contains the values of function $x^{*}(t)$, is contained within a ball $S_{R}$ of radius $R$. For vectors $\xi$ and $\eta$ from $S_{R}$ Lipschitz inequality takes place

$$
|\varphi(\xi)-\varphi(\eta)| \leq l|\xi-\eta|,
$$

where the positive number $l$ satisfies the condition

$$
l<1 /(2 T) .
$$

3) There can be found a positive number $M$, such that 
for all vectors $x$ from $S_{R}$ takes place

$$
|f(t, x)| \leq M, r+2 T M \leq R .
$$

\section{The Transition to Auxiliary System of Equations}

To simplify the notation let's assume that initial time moment is zero. Let's rewrite (1) in the integral form

$$
x(t)=C+\int_{0}^{t} f(\tau, x(\tau)) \mathrm{d} \tau,
$$

where vector $C$ defines the initial conditions. So far as $x^{*}(t)-T$-periodic function then

$$
x^{*}(0)=x^{*}(T) \equiv C^{*} .
$$

Then

$$
\int_{0}^{T} f\left(\tau, x^{*}(\tau)\right) \mathrm{d} \tau=0
$$

Therefore

$$
\frac{1}{T} \int_{0}^{T} f\left(\tau, x^{*}(\tau)\right) \mathrm{d} \tau=0 .
$$

So far as function $x^{*}(t)$ satisfies the system (1), then it also satisfies the system

$$
\dot{y}=f(t, y)-\frac{1}{T} \int_{0}^{T} f(\tau, y(\tau)) \mathrm{d} \tau .
$$

Let's pass from Equation (7) to the integral relation:

$$
y(t)=C+\int_{0}^{t}\left[f(s, y(s))-\frac{1}{T} \int_{0}^{T} f(\tau, y(\tau)) \mathrm{d} \tau\right] \mathrm{d} s .
$$

Let's notice that transition to the auxiliary system (8) is necessary because the nested integral (mean integral value over the period) in calculations allows avoiding the appearance degrees of $t$ in symbolic expressions. This reduces the amount of memory allocated for them, and also forms a symbolic representation of the desired function as an approximation to the Fourier series of periodic solutions of system (1).

\section{Scheme of Successive Approximations}

To obtain an approximation to the solution $x^{*}(t)$ on the period, first let's construct an approximation to a function that is a solution to the system (8). Then by varying the vector $C$ let's find the desired approximation. To do this let's show that we are in terms of applicability of the method of successive approximations.

Let $\Omega$-space of continuous $T$-periodic vector functions $y(t)$ with values in a ball $S_{R}$. The distance between the functions $p, q \in \Omega$ we'll define as

$$
\rho(p, q)=\max _{t \in[0, T]}|p(t)-q(t)| .
$$

Thus space $\Omega$ is metric. In $\Omega$ let's consider an operator

$$
\begin{aligned}
\Phi y(t) & =C+\int_{0}^{t}\left[f(s, y(s))-\frac{1}{T} \int_{0}^{T} f(\tau, y(\tau)) \mathrm{d} \tau\right] \mathrm{d} s \\
& \equiv C+\int_{0}^{t} u(s) \mathrm{d} s .
\end{aligned}
$$

Let's show that function

$$
g(t)=\Phi y(t)
$$

belongs to space $\Omega$, when $y \in \Omega$.

By virtue of (9) and $|C| \leq r$ (we will search a vector $C^{*}$ in a ball $S_{r}$, because it contains values of a function $\left.x^{*}(t)\right)$ we obtain:

$$
|g(t)| \leq|C|+\left|\int_{0}^{t} u(s) \mathrm{d} s\right| \leq r+2 T \max _{t \in[0, T], y \in \Omega}|f(t, y(t))| .
$$

Given that $y(t)$ takes values from $S_{R}$, and inequality (5), we obtain

$$
|g(t)| \leq R
$$

for all $t \in[0, T]$.

Now let's show the $T$-periodicity of function $g(t)$ :

$$
g(t+T)-g(t)=0
$$

for any real values $t$. With (9) let's consider the difference

$$
\begin{aligned}
g(t+T)-g(t) & =\int_{0}^{t+T} u(s) \mathrm{d} s-\int_{0}^{t} u(s) \mathrm{d} s \\
& =\int_{0}^{t+T} u(s) \mathrm{d} s+\int_{t}^{0} u(s) \mathrm{d} s .
\end{aligned}
$$

Since the function $f T$-periodic in $t$ and $y(t)$ is also $T$-periodic then function $u(s)$ will be $T$-periodic. In view of this making the change $s=a+T$ in first integral we obtain

$$
\begin{aligned}
g(t+T)-g(t) & =\int_{-T}^{t} u(a) \mathrm{d} a+\int_{t}^{0} u(s) \mathrm{d} s \\
& =\int_{-T}^{0} u(s) \mathrm{d} s=\int_{0}^{T} u(s) \mathrm{d} s .
\end{aligned}
$$

Let's assume

$$
B=\int_{0}^{T} f(\tau, y(\tau)) \mathrm{d} \tau
$$

Then

$$
\begin{aligned}
g(t+T)-g(t) & =\int_{0}^{T}\left[f(s, y(s))-\frac{1}{T} B\right] \mathrm{d} s \\
& =B-\frac{1}{T} B T=0 .
\end{aligned}
$$


Thus if $y \in \Omega$ then $g \in \Omega$.

Let's show that mapping $\Phi y$ is contracted. Let's estimate

$$
\begin{aligned}
& \rho(\Phi p, \Phi q)=\max _{t \in[0, T]} \mid \int_{0}^{t}[f(s, p(s))-f(s, q(s)) \\
& \left.-\frac{1}{T} \int_{0}^{T}\{f(\tau, p(\tau))-f(\tau, q(\tau))\} \mathrm{d} \tau\right] \mathrm{d} s \mid
\end{aligned}
$$

By virtue of inequality (3) and (2) we obtain

$$
\rho(\Phi p, \Phi q) \leq 2 l T \rho(p, q) \text {. }
$$

From (4) it follows that

$$
2 l T<1 .
$$

Thus the mapping is a compression.

Then, according to the method of successive approximations the scheme

$$
y_{m}(t)=C+\int_{0}^{t}\left[f\left(s, y_{m-1}(s)\right)-\frac{1}{T} \int_{0}^{T} f\left(\tau, y_{m-1}(\tau)\right) \mathrm{d} \tau\right] \mathrm{d} s
$$

converges to solution of a system (8).

\section{Finding Periodic Solution}

Since the right part of the system (1) on $x$ a multidimensional polynomial and in $t$ it is a trigonometric polynomial the initial function $y_{0}(t)$ it is advisable to choose equal to vector $C$ or as

$$
y_{0}(t)=C \cos (\omega t),
$$

where $\omega=2 \pi / T$, i.e., $y_{0} \in \Omega$.

Note that the multiplication of two (and, consequently, any number of) trigonometric functions (cos or $\sin$ ) and also an extent of such functions [4], can be represented as sum of a constant vector and trigonometric polynomial, and during the integration of trigonometric polynomial is obtained the same sum. Then from the scheme (10), each iteration can be calculated symbolically. At that the transformation of trigonometric functions in symbolic form, as well as their symbolic integration, are parallelized. The idea of parallelism lies in the formula (10): calculations for each component of the vector $y_{m}(t)$ might be produced independently of each other and store the obtained symbolic expressions in network database which accessible for computational process in a distributed computing environment.

Following the formula (10), we build a function $y_{m}(t)$ to such value $m$, when

$$
\max _{C \in S_{r}} \rho\left(y_{m}, y_{m-1}\right)<\varepsilon_{c},
$$

where $\varepsilon_{c}$-accuracy for the scheme (10).

Let's consider the integral

$$
I=\int_{0}^{T} f(\tau, y(\tau)) \mathrm{d} \tau .
$$

When substituting a character expression for the function we obtain vector function from $C$. Then redenote

$$
\delta(C)=I .
$$

We need to find a vector $C$ such $\left(C=C^{*}\right)$ that there takes place an Equation (6), i.e.

$$
\delta\left(C^{*}\right)=0 .
$$

So far as the system (1) has a unique periodic solution then system of algebraic Equations (12) in region $S_{r}$ will also have a unique solution.

The system (12) is equivalent to

$$
\left\langle\delta\left(C^{*}\right), \delta\left(C^{*}\right)\right\rangle=0 .
$$

From where we get the optimization problem to find an approximate value of vector $C^{*}$ :

$$
\theta(C)=\langle\delta(C), \delta(C)\rangle \rightarrow \min , C \in S_{r} .
$$

The transition from the system (12) of algebraic equations to the problem (13) related to the fact that directly in the calculations the function $y(t)$ is defined approximately by using the criterion (11).

\section{An Example of a Nonlinear Second-Order System}

As an example of using the described method let's consider a nonlinear oscillations equation (in this subsection, we rename some functions, as is customary in well-known literature)

$$
\ddot{x}+q(x) \dot{x}+g(x)=p(t),
$$

where

$$
\begin{gathered}
q(x)=0.01\left(1+2 x^{2}\right), \\
g(x)=0.03 x, \\
p(t)=0.01 \cos (24 t),
\end{gathered}
$$

$T$ period of right part is equal to $\pi / 12$. According to Theorem 8.1 [2] for an Equation (14) the convergence property is executed.

From an Equation (14) let's move to an equivalent system of second order [2], where right part has the form (2):

$$
\dot{x}=y-Q(x)+P(t), \quad \dot{y}=-g(x),
$$

where

$$
Q(x)=\int_{0}^{x} q(\xi) \mathrm{d} \xi=0.01\left(x+\frac{2}{3} x^{3}\right),
$$




$$
P(t)=\int_{0}^{t} p(\tau) \mathrm{d} \tau=\frac{1}{2400} \sin (24 t) .
$$

Let's find the radius $r$ of the ball $S_{r}$, where all solutions of system (15) are bounded. To do this, let's first show the fulfillment of conditions 1 - 4 [2]:

1) Functions $q, g$ and $p$ are continuous, $g$ satisfies a Lipschitz condition with constant 0.03 .

2) The value of numbers $a, \alpha$ and $\beta$ is equal to $1 / 12$, $73 / 7200$ and $1 / 400$ respectively so that $q(x) \geq \alpha$ when $|x| \geq a, \quad g(x) \geq \beta \quad$ when $x \geq a, \quad g(x) \leq-\beta \quad$ when $x \leq-a$.

3) A function $P(t)$ is bounded at all $t$, i.e., $|P(t)|<E, \quad E=1 / 1200$.

4) There exists such number $\gamma=1 / 259,200$, that

$$
Q(x)-E \geq \gamma
$$

when $x \geq a$,

$$
Q(x)+E \leq-\gamma
$$

when $x \leq-a ; G(x)>0$ when $|x| \geq a$, where

$$
G(x)=\int_{0}^{x} g(\xi) \mathrm{d} \xi=0.015 x^{2} .
$$

Then any solution of system (15) is bounded in a rectangle, defined by inequalities

$$
|x| \leq a,|y| \leq \lambda_{0}+\lambda_{1} \equiv b,
$$

where $\lambda_{0}$ so that $|Q(x)|+E \leq \lambda_{0}$ when $|x| \leq a$. Let's choose it as equal to

$$
\lambda_{0}=\max _{|x| \leq a}|Q(x)|+E .
$$

Since $Q(x)$-everywhere increasing function then $\lambda_{0}=\gamma$. The value of constant $\lambda_{1}$ is chosen as [2]

$$
\lambda_{1}=\sqrt{\lambda_{0} a \frac{\delta}{\gamma}}+\Delta,
$$

where $\Delta$-any positive number and

$$
\delta=\max _{|x| \leq a}|g(x)|[2] .
$$

Let's assume $\Delta=\sqrt{3} / 1200-\gamma$. Then

$$
b=\sqrt{3} / 600 \text {. }
$$

Thus, radius $r$ may be taken equal to

$$
r=\sqrt{a^{2}+b^{2}}=\sqrt{7} / 30 .
$$

Now let's establish the fulfillment of conditions 3 and 2 from section 2 of given article. Let's rewrite the system (15) in a vector form:

$$
\dot{z}=w(t, z),
$$

where $z(t)=(x(t), y(t))$. Let's consider a ball $S_{R}$ of radius

$$
R=0.5
$$

which have inside a ball $S_{r}$. Then from (15)

$$
\begin{aligned}
& r+2 T \max _{t \in[0, T], z \in \Omega}|w(t, z(t))| \\
& \leq r+2 T \max _{|x| \leq R,|y| \leq R}\left[|y|+0.01\left(|x|+\frac{2}{3}|x|^{3}\right)+E+0.03|x|\right] \\
& \leq R .
\end{aligned}
$$

Local implementation of the Lipschitz condition for the function $\varphi(z)$ is established the following way. Let's represent

$$
\varphi\left(z_{1}\right)-\varphi\left(z_{2}\right)=A\left(x_{1}, x_{2}\right)\left(z_{1}-z_{2}\right),
$$

where the $A$ matrix has the form

$$
A\left(x_{1}, x_{2}\right)=\left[\begin{array}{cc}
-0.01\left(1+\frac{2}{3}\left\{x_{1}^{2}+x_{1} x_{2}+x_{2}^{2}\right\}\right) & 1 \\
-0.03 & 0
\end{array}\right] .
$$

Hence we obtain

$$
2 T \max _{\left|x_{1}\right| \leq R,\left|x_{2}\right| \leq R}\left\|A\left(x_{1}, x_{2}\right)\right\|<1 .
$$

\section{Acknowledgements}

This article was supported by the Russian Foundation for Basic Research (Projects No. 11-07-00098 and 13-0700077).

\section{REFERENCES}

[1] M. A. Krasnosel'skij, "The Shift Operator along Trajectories of Differential Equations (in Russian),” Nauka, Moscow, 1966.

[2] V. A. Pliss, "Nonlocal Problems of Oscillation Theory (in Russian),” Nauka, Moscow, 1964, pp. 107-110,113.

[3] B. P. Demidovich, "Lectures on the Mathematical Stability Theory (in Russian),” Nauka, Moscow, 1967.

[4] I. S. Gradshtejn and I. M. Ryzhik, “Tables of Integrals, Sums, Series and Multiplications (in Russian),” Fizmatlit, Moscow, 1963, p. 39. 